\title{
Características químicas e salino-sodicidade dos solos do Perímetro Irrigado Califórnia, SE, Brasil
}

\author{
Characteristcs chemical and soil salinization in the Irrigated District of California, SE, Brazil
}

\author{
Antenor de Oliveira Aguiar Netto ${ }^{\mathrm{I}}$ Carlos Clériston Santana Gomes ${ }^{\mathrm{II}}$ Carla Cristina Valois Lins ${ }^{\mathrm{II}}$ \\ Allan Cunha Barros ${ }^{\mathrm{II}}$ Luis Fernando de Souza Magno Campeche ${ }^{\mathrm{III}}$ Flávio Favaro Blanco ${ }^{\mathrm{IV}}$
}

\section{RESUMO}

O manejo inadequado da irrigação pode resultar no acúmulo de sais no solo, cuja intensidade depende da qualidade da água utilizada na irrigação, da fração de lixiviação adotada e da demanda evapotranspirativa da região. O presente trabalho teve como objetivo avaliar os atributos físicos e químicos dos solos do perímetro irrigado Califórnia, localizado no extremo noroeste do Estado de Sergipe. Para tal, foram realizadas amostragens em 42 lotes, correspondendo a 16,4\% dos lotes deste perímetro. As maiores concentrações de sais e de sódio ocorreram na camada superficial $(0-0,2 m)$ do solo, o que evidenciou a falta de um manejo da irrigação adequado visando ao controle da salinidade. O aumento do $\mathrm{pH}$ do solo foi correlacionado significativamente com a porcentagem de sódio trocável, atingindo valores acima de 9,5. Correlações positivas significativas foram observadas entre a condutividade elétrica e os teores de Ca e $\mathrm{Mg}$, indicando que sais desses íons podem estar se acumulando no solo pela ascensão do lençol freático, associado à ausência de lixiviação e drenagem.

Palavras-chave: $\begin{aligned} & \text { salinidade, condutividade elétrica, } \\ & \text { sodicidade. }\end{aligned}$

\section{ABSTRACT}

The improper irrigation management may result in the accumulation of salts in the soil profile, which intensity depends on the irrigation water quality, the leaching fraction adopted and on the evapotranspiration demand of the region. The objective of the present study was to study the physical and chemical characteristics of the soils in the irrigated district of California, located in the northwest of the State of Sergipe.
Thus, soil sampling were made in 54 lots, corresponding to $16.4 \%$ of the lots of this district. The highest salt and sodium concentrations occurred in the shallowest soil layer $(0-0.2 \mathrm{~m})$, evidencing the lack of an appropriate irrigation management in order to control the salinity build up. The increasing of the soil $\mathrm{pH}$ was significantly correlated to the exchangeable sodium percentage, reaching values over 9.5. Significant positive correlations were observed between the electrical conductivity and the Ca and $\mathrm{Mg}$ content, indicating that salts of both ions could be accumulating in soil by the capillary rise of the water table, associated to the lack of leaching and drainage.

Key words: salinity, electrical conductivity, sodicity.

\section{INTRODUÇÃo}

Em áreas de elevada demanda evapotranspirativa e reduzido índice pluviométrico, a irrigação é prática obrigatória para a maioria dos cultivos. Este é o caso da região do semi-árido nordestino, onde a demanda de água pelas culturas não é atendida somente pelas chuvas, havendo, portanto, necessidade imprescindível de irrigação para que as áreas de produção sejam economicamente viáveis.

No Nordeste brasileiro, os solos afetados por sais naturalmente ocorrem em condições topográficas que favorecem a drenagem deficiente e, muitas vezes, a indução da salinidade decorre da

\footnotetext{
IDepartamento de Engenharia Agronômica, Universidade Federal de Sergipe (UFS), 49100-00, São Cristóvão, SE, Brasil. E-mail: antenor@ufs.br. Autor para correspondência.

IUFS, São Cristóvão, SE, Brasil.

IIICentro Federal de Educação Tecnológica (CEFET), Petrolina, PE, Brasil.

${ }^{\mathrm{IV}}$ Empresa Brasileira de Pesquisa Agropecuária (Embrapa Meio-Norte), Teresina, PI, Brasil.
} 
irrigação malconduzida e/ou com águas de qualidade duvidosa (OLIVEIRA, 1997). Um dos inconvenientes da irrigação é salinizar o solo. Isso ocorre pelo fato de a água de irrigação apresentar sais dissolvidos que, mesmo em baixa concentração, podem ser incorporados ao solo, o qual pode se tornar salino em poucos anos (MEDEIROS, 2001).

Cerca de $28 \%$ dos perímetros irrigados no Nordeste apresentam problemas de salinidade (GOES, 1978). Foi estimada por SZABOLCS (1989) em mais de 4 milhões de hectares a área de solos afetados por sais no Brasil. De acordo com SUASSUNA \& AUDRY (1993), o percentual de áreas irrigadas com problemas de salinização nesta região é de aproximadamente 32\%. Já SILVA (1997) afirma que aproximadamente 25\% da área irrigada encontra-se com problemas de salinidade e esta porcentagem poderá aumentar caso não sejam adotadas medidas preventivas urgentes. A salinização secundária, causada pela ação do homem, manifestase, principalmente, em decorrência da irrigação, em áreas onde o controle da drenagem não é realizado ou feito de forma ineficiente. No Nordeste semi-árido, as maiores incidências de áreas salinizadas com salinização secundária se concentram nas terras mais intensamente cultivadas com o uso da irrigação, nos chamados perímetros irrigados (OLIVEIRA, 1997). A salinização do solo tem como conseqüência a redução do rendimento dos cultivos, tornando necessário realizar uma lavagem de recuperação e adição de condicionadores químicos, como o gesso agrícola, o que leva a custos adicionais.

O presente trabalho tem como objetivo identificar os atributos físicos e químicos dos solos do perímetro irrigado Califórnia, localizado no extremo noroeste do Estado de Sergipe, bem como relacionar esses atributos a problemas de salino-sodicidade do solo.

\section{MATERIAL E MÉTODOS}

O Perímetro Irrigado Califórnia situa-se entre os municípios de Canindé do São Francisco e Poço Redondo, no extremo noroeste do Estado de Sergipe, microrregião homogênea Sertão Sergipano do São Francisco, distando $213 \mathrm{~km}$ de Aracaju. Nas áreas irrigáveis desse perímetro, utiliza-se a tecnologia de irrigação por aspersão, sendo o sistema alimentado com água captada diretamente do Rio São Francisco.

De acordo com o projeto original, deveriam coexistir em 3.980 hectares, de maneira integrada, interdependente e complementar, as duas formas de exploração agrícola recomendadas para a região semiárida: a agricultura irrigada e de sequeiro. Nas terras passíveis de irrigação, seria utilizada a técnica de irrigação por aspersão em 1.360ha, nos 2.620ha das terras não-irrigáveis, seriam implantados lotes de sequeiro, sendo que 650 hectares seriam utilizados para reservas florestais e 114ha ficariam para atividades de infra-estrutura, tais como estradas, casas, canais de condução de água, entre outros.

O clima é classificado como mediterrâneo quente ou nordestino, de seca acentuada no verão ou muito quente, semi-árido, tipo estepe, com estação chuvosa no inverno. A pluviosidade média na área é de 483,9mm ano ${ }^{-1}$, com precipitação máxima anual de 918,6 e mínima de 203,0mm ano-1. A classificação dos solos predominantes na área, em ordem decrescente de área ocupada, é a seguinte, de acordo com COHIDRO (2001): Luvissolo, Neossolo Litólico eutrófico, Vertissolos, Cambissolos Eutróficos e Argissolo Vermelho-Amarelo eutrófico.

Realizou-se uma amostragem probabilística intencional, sendo escolhido um determinado número de lotes, cujas amostras foram coletadas de acordo com a identificação visual e indicação, pelos técnicos do perímetro, de processos avançados de comprometimento dos solos pela acumulação de elevados teores de sais na superfície ou em lotes próximos. O levantamento das características químicas e físicas dos solos de 16,4\% dos lotes, num total de 42 lotes, foi realizado com o intuito de estabelecer uma amostra representativa do universo de lotes do Perímetro.

As amostras para a determinação das características químicas e físicas foram em número de 84 e com massa aproximada de $0,5 \mathrm{~kg}$, em média. Estas foram coletadas com trado, sendo retiradas nas profundidades de 0,0-0,2m e 0,2-0,4m. Após a coleta, as amostras foram acondicionadas em sacos plásticos, etiquetadas e encaminhadas para os laboratórios de química ambiental e de água e solo da UFS, para fins de análise física e química. Na análise da salinização, foram determinadas as condutividades elétricas (CE), com auxílio de um condutivímetro, e a porcentagem de sódio trocável (PST). Os valores de pH e de cálcio (Ca), magnésio $(\mathrm{Mg})$, potássio $(\mathrm{K})$ e sódio $(\mathrm{Na})$ trocáveis foram determinados de acordo com EMBRAPA (1998). Para a avaliação e classificação dos solos afetados por sais, considerou-se o que preconiza o Laboratório de Salinidade dos Estados Unidos (RICHARDS, 1954), baseada no pH em água, CE e PST.

A fim de se realizar uma avaliação inicial das relações existentes entre as características físicas e químicas do solo, foram obtidas matrizes de correlação linear para todas as variáveis analisadas. A análise foi realizada, inicialmente, utilizando-se a média das amostragens nas duas profundidades e, 
posteriormente, para cada profundidade, de modo que o efeito desta variável pudesse ser mais bem avaliado.

\section{RESULTADOS E DISCUSSÃO}

A tabela 1 mostra a classificação dos solos estudados no Perímetro Irrigado Califórnia quanto à presença de sais, baseada na condutividade elétrica e na porcentagem de sódio trocável. As maiores concentrações de sais e de sódio ocorreram na camada superficial do solo (0-0,2m), o que evidenciou a falta de um manejo da irrigação adequado visando ao controle da salinidade, com predominância de solos salinosódicos.

A condutividade elétrica expressa a concentração de sais solúveis no solo. KLAR (1988) afirma que as culturas respondem diferentemente às concentrações salinas, podendo ser quantificadas de acordo com os valores dessa condutividade. Segundo esse autor, os efeitos da salinidade para as plantas são negligenciáveis entre 0 e $2 \mathrm{dS} \mathrm{m}{ }^{-1}$; de 2 a $4 \mathrm{dS} \mathrm{m}^{-1}$, pode ocorrer restrição do desenvolvimento de algumas culturas; de 4 a $8 \mathrm{dS} \mathrm{m}^{-1}$, muitas culturas diminuem a produção; de 8 a $16 \mathrm{dS} \mathrm{m} \mathrm{m}^{-1}$, só algumas plantas produzem satisfatoriamente e, acima de $16 \mathrm{dS} \mathrm{m} \mathrm{m}^{-1}$, somente as culturas tolerantes produzem satisfatoriamente.

Do lote de amostras analisadas para ambas as profundidades amostradas, 64\% apresentaram valores de condutividade elétrica acima de $2 \mathrm{dS} \mathrm{m}^{-1}$, o que caracteriza risco iminente para a produção satisfatória das culturas, enquanto 54\% das amostras apresentaram porcentagem de sódio trocável acima de 15. Quando isso ocorre, a estrutura de muitos solos deteriora-se rapidamente, conforme demonstrado pelos resultados de MARCIANO et al. (2000) e AGUIAR NETTO et al. (2005). Os solos salinos podem ser facilmente identificados em condições naturais pela presença de uma crosta branca e por encontrarem-se normalmente floculados, devido à presença de sais em excesso, associada à ausência de quantidades significativas de sódio trocável (GHEYI, 2000).

Na tabela 2, é apresentada a matriz de correlação linear obtida para o perfil de solo, enquanto na tabela 3 encontra-se a matriz de correlação para cada profundidade estudada. Verifica-se que a profundidade de amostragem apresentou relação inversa com a Porcentagem de Sódio Trocável (PST), equivalente a -0,34*, e com o teor de Sódio ( $\mathrm{Na}$ ), igual a -0,40*, o que indica maior concentração deste elemento na camada superficial do solo, onde se concentra o sistema radicular das culturas. Além disso, esse fato é um indicativo de que o manejo da irrigação nessas áreas não vem sendo realizado de forma a evitar o acúmulo de sais na profundidade efetiva do sistema radicular das plantas, uma vez que a aplicação de uma fração de lixiviação deveria resultar em maior acúmulo de sais nas camadas mais profundas de solo. Apesar de não ter havido relação significativa entre CE e a profundidade, os valores médios foram de 3,9 e 2,9dS $\mathrm{m}^{-1}$ para as profundidades de $0-0,2 \mathrm{~m}$ e de $0,2-0,4 \mathrm{~m}$, respectivamente, corroborando esta afirmação.

O aumento da PST resultou em um significativo aumento do $\mathrm{pH}$ do solo, somente na profundidade $0-0,2 \mathrm{~m}$ (Tabela 3), na qual o valor do $\mathrm{pH}$ chegou a 9,5 e o PST a 57 mmol $_{\mathrm{c}} \mathrm{L}^{-1}$ (Figura 1a). Sob condições de $\mathrm{pH}$ elevado, ocorre a mudança de cargas líquidas das argilas, que passam de positivas para negativas; com isso, ocorre um aumento no valor da floculação do solo, o que resulta num aumento da dispersão das argilas (RICHARDS, 1954), reduzindo a permeabilidade do solo (SHAINBERG \& LETEY, 1984). Além disso, a elevação do $\mathrm{pH}$ resulta na alteração da

Tabela 1 - Classificação dos solos quanto à salinidade baseada na condutividade elétrica e na porcentagem de sódio trocável dos lotes amostrados no Perímetro Irrigado Califórnia.

\begin{tabular}{|c|c|c|}
\hline Quantidade de lotes & Porcentagem (\%) & Classificação \\
\hline \multicolumn{3}{|c|}{ Profundidade $0,0-0,2 \mathrm{~m}$} \\
\hline 6 & 14 & Normal $^{1}$ \\
\hline 8 & 19 & Salino $^{2}$ \\
\hline 9 & 22 & Sódico $^{3}$ \\
\hline 19 & 45 & Salino-sódico ${ }^{4}$ \\
\hline \multicolumn{3}{|c|}{ Profundidade $0,2-0,4 \mathrm{~m}$} \\
\hline 21 & 50 & Normal \\
\hline 6 & 14 & Salino \\
\hline 6 & 14 & Sódico \\
\hline 9 & 22 & Salino-sódico \\
\hline
\end{tabular}

1: fora das faixas de salino-sodicidade; 2 : $\mathrm{pH}<8,5$ - $\mathrm{CE}>4 \mathrm{dS} / \mathrm{m}-\mathrm{PST}<15$; 3: $\mathrm{pH}>8,5-\mathrm{CE}<4 \mathrm{dS} / \mathrm{m}-\mathrm{PST}>15$; 4 : $\mathrm{pH}<8,5-\mathrm{CE}>4$ $\mathrm{dS} / \mathrm{m}-\mathrm{PST}>15$. 
Tabela 2 - Matriz de correlação linear das características físicas e químicas dos solos avaliados, para todo o perfil do solo.

\begin{tabular}{|c|c|c|c|c|c|c|c|c|c|c|c|}
\hline Característica & Profundidade & Areia & Silte & Argila & $\mathrm{pH}$ & $\mathrm{CE}$ & PST & $\mathrm{P}$ & $\mathrm{Ca}$ & $\mathrm{Mg}$ & K \\
\hline & \multicolumn{11}{|c|}{ Profundidade $=0-0,4 \mathrm{~m}$} \\
\hline Areia & $-0,09$ & & & & & & & & & & \\
\hline Silte & 0,12 & $-0,96^{*}$ & & & & & & & & & \\
\hline Argila & $-0,15$ & $-0,01$ & $-0,26^{*}$ & & & & & & & & \\
\hline $\mathrm{pH}$ & $-0,01$ & $-0,12$ & 0,16 & $-0,17$ & & & & & & & \\
\hline Condutividade elétrica (CE) & $-0,16$ & 0,10 & $-0,20$ & $0,36 *$ & $-0,48^{*}$ & & & & & & \\
\hline Porcentagem de sódio trocável (PST) & $-0,34 *$ & 0,17 & $-0,13$ & $-0,12$ & $0,42 *$ & 0,04 & & & & & \\
\hline $\mathrm{P}$ & 0,06 & 0,11 & $-0,09$ & $-0,06$ & 0,10 & $-0,06$ & $-0,02$ & & & & \\
\hline $\mathrm{Ca}$ & $-0,10$ & $-0,20$ & 0,09 & $0,40 *$ & $-0,36^{*}$ & $0,29 *$ & $-0,45^{*}$ & $-0,01$ & & & \\
\hline Mg & $-0,10$ & $-0,20$ & 0,08 & $0,41 *$ & $-0,36^{*}$ & $0,29 *$ & $-0,44^{*}$ & 0,00 & $0,96 *$ & & \\
\hline $\mathrm{K}$ & $-0,19$ & 0,03 & $-0,04$ & 0,03 & 0,14 & 0,06 & $0,36^{*}$ & $0,35 *$ & 0,11 & 0,13 & \\
\hline $\mathrm{Na}$ & $-0,40^{*}$ & 0,06 & $-0,04$ & $-0,08$ & $0,38^{*}$ & 0,08 & $0,85^{*}$ & 0,07 & $-0,14$ & $-0,12$ & $0,64^{*}$ \\
\hline
\end{tabular}

disponibilidade de nutrientes no solo (BAILEY, 1996), o que pode levar à deficiência ou ao excesso de determinados macro ou micronutrientes nas plantas.

A correlação entre $\mathrm{Ca}$ e $\mathrm{Mg}$ foi de, aproximadamente, 1:1 (Figura 1b). Observou-se correlação significativa, em nível de 0,05 de probabilidade, entre o $\mathrm{pH}$ e os teores de $\mathrm{Ca}$ e $\mathrm{Mg}$ no solo, sendo que a redução dos teores desses elementos esteve correlacionada à redução do $\mathrm{pH}$ (Tabela 2), o que não era esperado. Entretanto, a correlação positiva entre a CE e o Ca e o Mg é um indicativo de que os sais desses elementos são, em parte, responsáveis pela elevação da salinidade do solo na camada $0-0,2 \mathrm{~m}$ de profundidade. $\mathrm{O}$ excesso desses nutrientes pode ser devido à superaplicação de fertilizantes e/ou calcário, ou ainda à qualidade da água do lençol freático. FERREIRA (1997) afirma que, quando águas freáticas se aproximam da superfície do solo, a

Tabela 3 - Matrizes de correlação simples das características físicas e químicas dos solos avaliados, para cada profundidade de amostragem.

\begin{tabular}{|c|c|c|c|c|c|c|c|c|c|c|}
\hline Característica & Areia & Silte & Argila & $\mathrm{pH}$ & $\mathrm{CE}$ & PST & $\mathrm{P}$ & $\mathrm{Ca}$ & $\mathrm{Mg}$ & $\mathrm{K}$ \\
\hline \multicolumn{11}{|c|}{ Profundidade 0,0 - 0,2 m } \\
\hline Silte & $-0,93 *$ & & & & & & & & & \\
\hline Argila & 0,01 & $-0,38 *$ & & & & & & & & \\
\hline $\mathrm{pH}$ & $-0,17$ & 0,24 & $-0,22$ & & & & & & & \\
\hline Condutividade elérica (CE) & 0,14 & $-0,29 *$ & $0,40 *$ & $-0,51^{*}$ & & & & & & \\
\hline Porcentagem de sódio trocável (PST) & 0,02 & 0,07 & $-0,23$ & $0,66^{*}$ & $-0,11$ & & & & & \\
\hline $\mathrm{P}$ & 0,17 & $-0,13$ & $-0,08$ & 0,08 & $-0,08$ & $-0,28$ & & & & \\
\hline $\mathrm{Ca}$ & $-0,18$ & 0,00 & $0,46^{*}$ & $-0,50 *$ & $0,38 *$ & $-0,64^{*}$ & 0,09 & & & \\
\hline $\mathrm{Mg}$ & $-0,21$ & 0,01 & $0,49 *$ & $-0,50 *$ & $0,39 *$ & $-0,62 *$ & 0,08 & $0,96 *$ & & \\
\hline K & $-0,20$ & 0,17 & 0,03 & 0,14 & $-0,13$ & 0,08 & $0,29 *$ & 0,14 & 0,22 & \\
\hline $\mathrm{Na}$ & $-0,18$ & 0,24 & $-0,19$ & $0,56^{*}$ & $-0,08$ & $0,83^{*}$ & $-0,20$ & $-0,30 *$ & $-0,26$ & $0,34 *$ \\
\hline \multicolumn{11}{|c|}{ Profundidade 0,2 - 0,4 m } \\
\hline Silte & $-1,00 *$ & & & & & & & & & \\
\hline Argila & $-0,17$ & 0,10 & & & & & & & & \\
\hline $\mathrm{pH}$ & $-0,07$ & 0,08 & $-0,04$ & & & & & & & \\
\hline $\mathrm{CE}$ & 0,01 & $-0,02$ & $0,15^{\mathrm{ns}}$ & $-0,46^{*}$ & & & & & & \\
\hline PST & $0,31^{*}$ & $-0,30$ & $-0,14$ & $0,20^{\mathrm{ns}}$ & 0,17 & & & & & \\
\hline $\mathrm{P}$ & 0,09 & $-0,09$ & $-0,06$ & 0,13 & $-0,05$ & 0,19 & & & & \\
\hline $\mathrm{Ca}$ & $-0,24$ & 0,21 & $0,42 *$ & $-0,19$ & $0,13^{\text {ns }}$ & $-0,36 *$ & $-0,06$ & & & \\
\hline $\mathrm{Mg}$ & $-0,22$ & 0,20 & $0,39 *$ & $-0,21$ & $0,09^{\mathrm{ns}}$ & $-0,36^{*}$ & $-0,04$ & $0,98 *$ & & \\
\hline $\mathrm{K}$ & 0,12 & $-0,12$ & $-0,10$ & 0,17 & 0,18 & $0,52 *$ & $0,40^{*}$ & 0,08 & 0,05 & \\
\hline $\mathrm{Na}$ & 0,23 & $-0,22$ & $-0,16$ & 0,23 & 0,19 & $0,84^{*}$ & 0,27 & $-0,09$ & $-0,09$ & $0,84^{*}$ \\
\hline
\end{tabular}

* Significativo a 0,05 de probabilidade pelo teste t-Student; ns: não-significativo. 

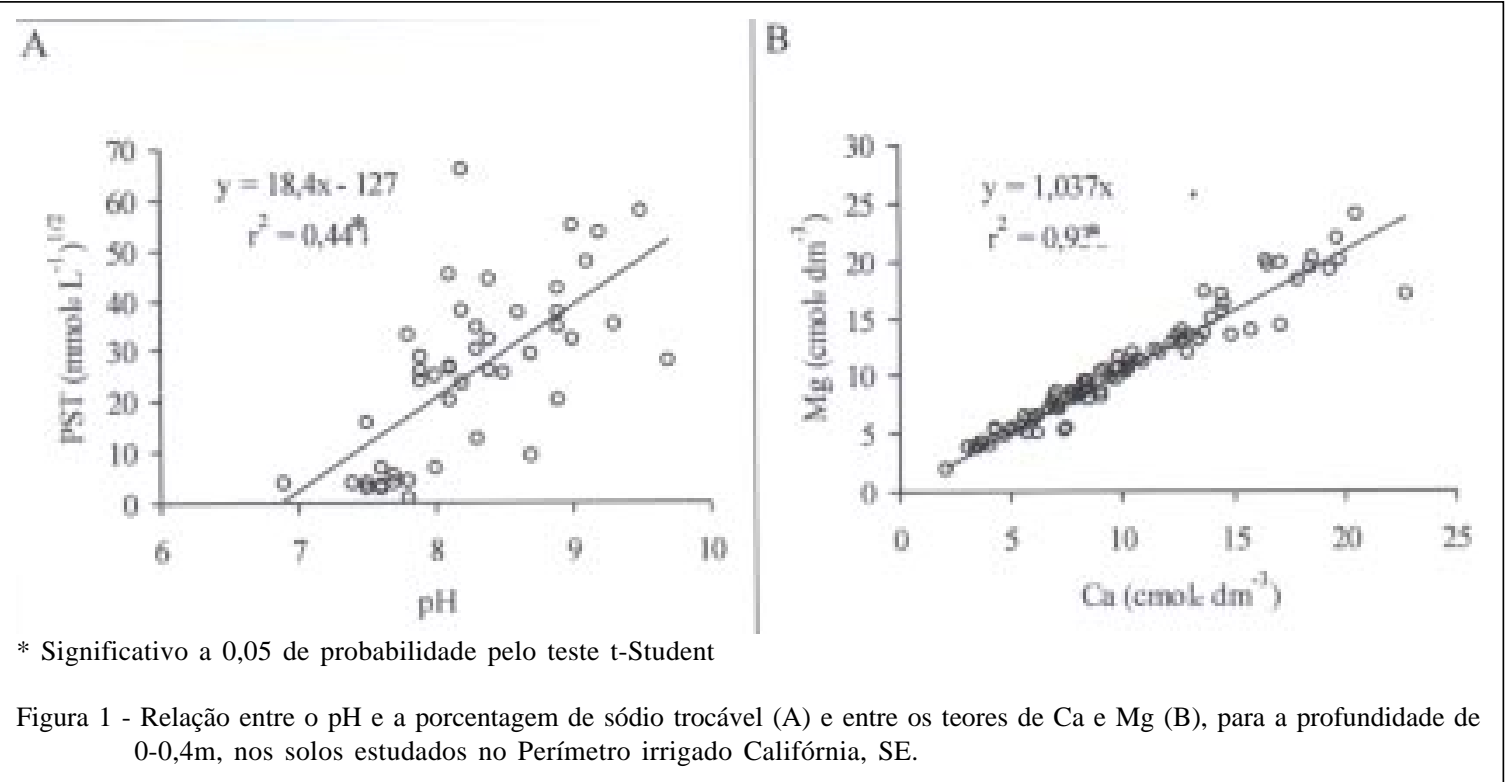

evapotranspiração provoca a concentração de carbonato de Ca e de Mg no perfil. O teor de argila no solo teve correlação positiva com a CE e com os teores de Ca e Mg (tabela 2), corroborando a afirmação anterior, haja vista que, na ausência de lixiviação, o acúmulo de cátions nas argilas da camada superficial de solo é progressivo.

O excesso de sais em solos do Perímetro Irrigado Califórnia é possivelmente uma conseqüência do manejo inadequado, pelo uso exagerado de água na irrigação, conforme salientado por técnicos do perímetro. Outro aspecto que pode estar relacionado com esse excesso de sais é o assoreamento dos drenos naturais nos talvegues naturais, os quais promovem o escoamento do excesso de água da irrigação e das chuvas. Isso revela a necessidade de mudança de comportamento dos agricultores quanto ao manejo da irrigação e do solo.

Para evitar ou minimizar o avanço da área salinizada no Perímetro Irrigado Califórnia, devem-se tomar medidas imediatas, como a redução da quantidade de água aplicada na irrigação, utilizar a rotação de culturas e mesmo, em algumas áreas críticas, usar o gesso. A longo prazo, o órgão gestor dessa área irrigada deve efetuar estudos de drenagem agrícola e realizar a troca dos emissores, priorizando aspersores com reduzida intensidade de aplicação de água.

\section{CONCLUSÕES}

As análises realizadas nos solos do Perímetro Irrigado Califórnia mostraram que a maior parte dos solos encontra-se com problemas de salinosodicidade, caracterizando risco de queda na produção das culturas, devendo-se adotar medidas imediatas de manejo da água e do solo para impedir o avanço das áreas salinizadas.

\section{REFERÊNCIAS}

AGUiAR NETTO, A.O. et al. Diagnóstico do processo de salino-sodificação no perímetro irrigado Jabiberi-Se. In: CONGRESSO NACIONAL DE IRRIGAÇÃO E DRENAGEM, 15., 2005, Teresina. Anais... Teresina: Embrapa Meio Norte, 2005. V.1, p.1-6. (CD-ROM).

BAILEY, D.A. Alkalinity, $\mathrm{pH}$ and acidification. In: REED, D.W. Water, media, and nutrition for greenhouse crops. Batavia: Ball Publishing, 1996. Cap.4, p.69-91.

COHIDRO. Relatório 2001. Aracaju, 2001. 34p.

EMBRAPA. Manual de métodos de análise de solo. Rio de Janeiro: Serviço Nacional de Levantamento e Conservação de Solos, 1998. 212p.

FERREIRA, P.A. Aspectos físico-químicos do solo. In: GHEYI, H.R. et al. Manejo e controle da salinidade na agricultura irrigada. Campina Grande: UFPB/SBEA, 1997. Cap.2, p.37-67.

GHEYI, H.R. Problemas de salinidade na agricultura irrigada. In: OLIVEIRA et al. (Orgs.). Agricultura, sustentabilidade e o semi-árido. Fortaleza: UFS/SBCS, 2000. p.123-145.

GOES, E.S. de. O problema de salinidade e drenagem em projetos de irrigação do Nordeste e a ação da pesquisa com vistas a seu equacionamento. In: REUNIÃO SOBRE SALINIDADE EM 
ÁREAS IRRIGADAS, Fortaleza, 1978. Anais... Recife: Ministério do Interior/SUDENE, 1978. p.89-91.

KLAR, A.E. A água no sistema solo-planta-atmosfera. 2.ed. São Paulo: Nobel, 1988. 408p.

MARCIANO, C.R. et al. Infiltração da água em luvissolo salinizado. In: REUNIÃO BRASILEIRA DE MANEJO E CONSERVAÇÃO DO SOLO E DA ÁGUA, 13., 2000, Ilhéus. Resumos... Ilhéus: CEPLAC/SBCS, 2000. p.449-450.

MEDEIROS, J.F. Salinização em áreas irrigadas: manejo e controle. In: FOLEGATTI, M.V. et al. Fertirrigação: flores, frutas e hortaliças. Guaíba: Agropecuária, 2001. V.2, cap.2, p.201-240.

OLIVEIRA, M. Gênese, classificação e extensão de solos afetados por sais. In: GHEYI, H.R. et al. Manejo e controle da salinidade na agricultura irrigada. Campina Grande: UFPB/SBEA, 1997. Cap.1, p.1-35.
RICHARDS, L.A. Diagnosis and improvement of saline and alkali soils. Washington: USSL, 1954. 160p. (USDA. Agriculture Handbook, 60).

SHAINBERG, I.; LETEY, J. Response of soils to sodic and saline conditions. Hilgardia, v.52, n.2, p.1-57, 1984

SILVA, Ê.F. de F.E. Avaliação da eficiência de diversos produtos na recuperação de solo salino - sódico e no desenvolvimento e produção da cultura do arroz (Oriza sativa L.). 1997. 70f. Dissertação (Mestrado em Agronomia) - UFPB.

SUASSUNA, J.; AUDRY, P. Estatísticas de salinidade das águas de irrigação do Nordeste Semi-árido Brasileiro, ORSTOM/ FUNDAJ, Comunicação Oral. In: REUNIÃO ANUAL DA SBPC, 45., 1993, Recife. Anais ... Recife: Sociedade Brasileira para o Progresso da Ciência. 1993. 53p.

SZABOLCS, I. Salt-affected soils. Flórida: Library of Congress, 1989. 274p. 